NASA/CR-2000-210631

ICASE Report No. 2000-48

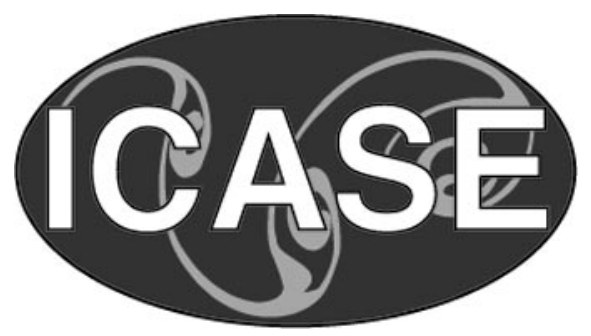

\title{
Mapping Closure Approximation to Conditional Dissipation Rate for Turbulent Scalar Mixing
}

Guowei He and R. Rubinstein

ICASE, Hampton, Virginia

ICASE

NASA Langley Research Center

Hampton, Virginia

Operated by Universities Space Research Association

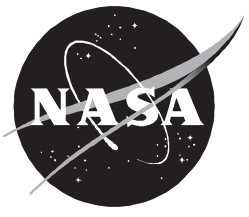

National Aeronautics and Space Administration 


\title{
MAPPING CLOSURE APPROXIMATION TO CONDITIONAL DISSIPATION RATE FOR TURBULENT SCALAR MIXING
}

\author{
GUOWEI HE* AND R. RUBINSTEIN ${ }^{\dagger}$
}

\begin{abstract}
A novel mapping closure approximation (MCA) technique is developed to construct a model for the conditional dissipation rate (CDR) of a scalar in homogeneous turbulence. It is shown that the CDR model from amplitude mapping closure is incorrect in asymptotic behavior for unsymmetric binary mixing. The correct asymptotic behavior can be described by the CDR model formulated by the MCA technique. The MCA approach is outlined for constructing successive approximation to probability density function (PDF) and conditional moment.
\end{abstract}

Key words. turbulent mixing, mapping closure, conditional dissipation rate, probability density function

Subject classification. Fluid Mechanics

1. Introduction. The probability density function (PDF) approach has been shown to be a useful tool in turbulence research. The systematic approach for determining the PDFs is by means of solving the transport equations for the PDFs. In the PDF equations for turbulent scalar fields, conditional dissipation rate (CDR) appears as the only unclosed term [1]. The recently developed large-eddy simulation schemes for turbulent reactive flow, such as filtered PDF approach [2], conditional moment closure [3] and Lagrangian flamelet model [4] also appeal the CDR models.

No satisfactory closure model for CDR had been constructed until the mapping closure approach $[5,6]$ was formulated. Amplitude mapping closure suggests a CDR model [7] whose form is separable in scalar and time variables. The model is in good agreement with direct numerical simulation (DNS) for initially symmetric binary mixing but fails in describing asymptotic behavior of the CDR for initially unsymmetric binary mixing [8]. Girimaji [9] has developed a novel amplitude mapping closure approach in which the reference fields are time-dependent. The CDR model obtained from a time-evolving Gaussian reference field still fails to describe the asymptotic behavior, but the one from a time-evolving Beta reference field can successfully describe the asymptotic behavior. This strongly suggests that the amplitude mapping closure is inadequate to describe the asymptotic behavior by itself.

In this paper, we will develop a novel mapping closure approximation (MCA) to make successive approximations to statistics of a scalar in homogeneous turbulence. This technique will be used to construct a CDR model which accounts for the asymptotic behavior of the CDR. In Section 2, we will investigate the asymptotic behavior of the CDR model from amplitude mapping closure and explain the reason why it fails to describe the asymptotic behavior correctly. In section 3, we will outline the MCA technique for successive approximation. In section 4, we will use the MCA technique to formulate a novel CDR model and compare it with DNS results. We will conclude with a summary in section 5 .

\footnotetext{
${ }^{*}$ ICASE, Mail Stop 132C, NASA Langley Research Center, Hampton, VA 23681-0001 (email:hgw@icase.edu). This research was supported by the National Aeronautics and Space Administration under NASA Contract No. NAS1-97046 while the author was in residence at ICASE, NASA Langley Research Center, Hampton, VA 23681.

†ICASE, Mail Stop 132C, NASA Langley Research Center, Hampton, VA 23681-0001 (email:bobr@icase.edu). This research was supported by the National Aeronautics and Space Administration under NASA Contract No. NAS1-97046 while the author was in residence at ICASE, NASA Langley Research Center, Hampton, VA 23681.
} 
2. Asymptotic behaviors of amplitude mapping closure. In this section, we will show that the CDR model from amplitude mapping closure has incorrect asymptotic behavior for unsymmetric binary mixing. We consider the simple case of a single conserved scalar $Z(x, t)$ in incompressible homogeneous and isotropic turbulence. With $u(x, t)$ being the velocity field and $\kappa$ the molecular diffusivity, the evolution equation for scalar $Z$ is

$$
\frac{\partial Z}{\partial t}+\mathbf{u} \cdot \nabla Z=\kappa \nabla^{2} Z
$$

Here the boundary condition is periodic and the scalar is initially homogeneous and isotropic. Therefore, the scalar $Z(x, t)$ will remain homogeneous and isotropic for all time. We further assume that the scalar is binary (0 or 1$)$ and its initial PDF is a double-delta distribution:

$$
P(Z, 0)= \begin{cases}A & \text { if } Z=0 \\ 1-A & \text { if } Z=1 \\ 0 & \text { if } Z \neq 0 \text { or } 1\end{cases}
$$

where $0 \leq A \leq 1 . A=0.5$ implies that the binary scalar has the same weights and the corresponding PDF $P(Z, 0)$ is symmetric with respect to $Z=0.5$, while $A \neq 0.5$ implies that the binary scalar has different weights and the corresponding $\operatorname{PDF} P(Z, 0)$ is unsymmetric. DNS [10] has shown that the scalar PDF $P(Z, t)$ asymptotically approaches a Gaussian distribution, whether the initial double-delta distribution is symmetric or unsymmetric. The mean $\langle Z\rangle=1-A$ remains unchanged in turbulent mixing. The CDR $\chi(Z, t)=\kappa\left\langle(\nabla Z)^{2} \mid Z\right\rangle$ is nearly parabolic. At early stages, the CDR maximum is located at $Z=0.5$, the mean of the initial interface of the binary scalar. The maximum then moves and finally approaches the mean $\langle Z\rangle$ of the scalar, accompanied by a distortion of the parabola. Therefore, for an initially symmetric binary scalar $(A=0.5)$, the maximum will remain fixed at $Z=0.5$, where the mean of the scalar and the mean of the initial interface are coincident. For an initially unsymmetric binary scalar $(A \neq 0.5)$, the maximum will shift to the mean $Z=1-A$ of the scalar from the mean $Z=0.5$ of the initial interface.

Amplitude mapping closure assumes

$$
Z=X\left(\phi_{0}, t\right)
$$

where $\phi_{0}(\mathbf{x})$ is a homogeneous Gaussian random field. The governing equation for the mapping function $X$ is

$$
\frac{\partial X}{\partial t}=\kappa\left\langle\phi_{0 x}^{2}\right\rangle\left(-\frac{\phi_{0}}{\left\langle\phi_{0}^{2}\right\rangle} \frac{\partial X}{\partial \phi_{0}}+\frac{\partial^{2} X}{\partial \phi_{0}^{2}}\right)
$$

where $\phi_{0 x}$ is the spatial gradient of the scalar $\phi_{0}$. The initial mapping function corresponding to (2.2) is

$$
X\left(\phi_{0}, 0\right)= \begin{cases}0 & \text { if } \phi_{0} \leq \gamma \\ 1 & \text { if } \phi_{0}>\gamma\end{cases}
$$

where $\gamma=\sqrt{2} \operatorname{erf}^{-1}(2 A-1)$. The exact solution of the equation (2.4) with the initial condition (2.5) is 


$$
X\left(\phi_{0}, t\right)=\frac{1}{2}\left[1+\operatorname{erf}\left(\frac{\theta_{0}-\gamma e^{\tau}}{\sqrt{2} \Sigma}\right)\right],
$$

where

$$
\begin{aligned}
& \theta_{0}=\frac{\phi_{0}}{\sqrt{\left\langle\phi_{0}^{2}\right\rangle}}, \\
& \tau=\kappa \frac{\left\langle\phi_{0 x}^{2}\right\rangle}{\left\langle\phi_{0}^{2}\right\rangle} t, \\
& \Sigma=\sqrt{\exp (2 \tau)-1} .
\end{aligned}
$$

We can calculate the CDR from the exact solution (2.6)

$$
\begin{aligned}
\chi(Z, t) & =\kappa \frac{\left\langle\phi_{0 x}^{2}\right\rangle}{\left\langle\phi_{0}^{2}\right\rangle} \frac{1}{\sqrt{2 \pi} \Sigma} \exp \left(-2\left[\operatorname{erf}^{-1}(2 Z-1)\right]^{2}\right) \\
& =\chi(0.5, t) \exp \left(-2\left[\operatorname{erf}^{-1}(2 Z-1)\right]^{2}\right) .
\end{aligned}
$$

The most striking feature of the CDR model (2.8) is the separability of its form in $Z$ and $t$. The separability suggests that the CDR's shape remains unchanged although its amplitude decays with time. The CDR maximum is located permanently at $Z=0.5$ without any shift for either symmetric or unsymmetric initial conditions. The permanent location of the maximum for unsymmetric initial condition $[7,8,9]$ is contrary to the known asymptotic behavior of the CDR. Therefore, the CDR model (2.8) from amplitude mapping closure is not able to describe the asymptotic behavior of the CDR. The amplitude mapping closure with a time-evolving Gaussian reference field gives a similar CDR model whose form is separable in $t$ and $Z$ and also fails to describe the shift of the maximum. Therefore, the separation in form is the main reason for the incorrect asymptotic behavior of the CDR model from amplitude mapping closure.

3. Mapping closure approximation. The basic idea of mapping closure is to represent an unknown random field by mapping of a known random reference field. The statistics of the unknown random field can be calculated from the mapping function and the known reference field. The governing equation for the mapping function can be obtained from the evolution equation of the unknown random field and the governing equation of its PDF.

Amplitude mapping closure assumes that the unknown random field can be mapped by a single known Gaussian reference field: $Z=X\left(\phi\left(x^{\prime}\right), t\right)$ and a coordinate transformation $d x^{\prime} / d x=J\left(d \phi\left(x^{\prime}\right) / d x^{\prime}, t\right)$ that accounts for turbulent stretching. The assumption [11] holds if and only if the spatial level crossing frequency at which the unknown random field passes through a given value has a single maximum as a function of that value. Therefore, the existence of the mapping function as well as the coordinate transformation is not ensured for arbitrary unknown random fields. Physically, a turbulent field exhibits eddies of different length and time scales so that it cannot be mapped by a single reference Gaussian field with a compact spectrum. Therefore, it is necessary to introduce more reference fields accounting for different eddies of different time and length scales:

$$
Z=X\left(\phi_{0}(x, t), \phi_{1}(x, t), \cdots, \phi_{n}(x, t) ; x, t\right)
$$


where $\phi_{i}, i=1, \cdots, n$, are reference fields. So far, we have not imposed any constraints on the reference fields so that we have some freedom in choosing the reference fields. For example, $\phi_{0}$ could be Gaussian but $\phi_{1}$ could be a Beta random field.

A one-to-one mapping could be established artificially between $\left(Z, \phi_{1}, \cdots, \phi_{n}\right)$ and $\left(\phi_{0}, \phi_{1}, \cdots, \phi_{n}\right)$. Thus, we can calculate the PDF and the conditional dissipation rate of the scalar

$$
\begin{aligned}
& P(Z ; x, t)=\int P\left(\phi_{0}, \phi_{1}, \cdots, \phi_{n}\right)\left(\frac{\partial X}{\partial \phi_{0}}\right)^{-1} d \phi_{1} \cdots d \phi_{n}, \\
& \left\langle(\nabla Z)^{2} \mid Z\right\rangle=\left\langle(\nabla X)^{2} \mid Z\right\rangle .
\end{aligned}
$$

Here the integration is taken over the entire subspace of the composition $\phi_{1}, \cdots, \phi_{n}$. The ensemble average is taken over the level surface on which $\left(\phi_{0}, \phi_{1}, \cdots, \phi_{n}\right)$ satisfies the constraint for a given $Z$

$$
X\left(\phi_{0}(x, t), \phi_{1}(x, t), \cdots, \phi_{n}(x, t) ; x, t\right)=Z \text {. }
$$

Introduction of more reference fields is expected to improve the approximation accuracy of mapping closure. The reference fields might be chosen to be statistically orthogonal so that more information can be introduced at less expenses. The hierarchy of mapping closure approximations with an increasing number of reference fields remains essentially non-perturbative. A perturbative approach, like the Wiener-Hermite expansion [13], could lead to the same result, but would require a prohibitively large number of terms to achieve the accuracy attainable with mapping closure based on a small number of reference fields.

4. The model for conditional dissipation rate. Broad classes of mapping function are admissible to MCA approach. The form to be considered here is

$$
Z=Y\left(\phi_{0}(x), \phi_{1}(t) ; t\right)
$$

where $\phi_{0}(x)$ is a homogeneous random Gaussian field in space and $\phi_{1}(t)$ an inhomogeneous random Gaussian field in time. Amplitude mapping closure requires $\phi_{1}(t)=0$, which fails in the asymptotic behavior of conditional dissipation rate due to lack of an independent time-evolving reference field.

Following the standard method [11, 12], we can formulate the mapping equation:

$$
\frac{\partial Y}{\partial t}+\frac{\partial Y}{\partial \phi_{1}}\left\langle\frac{d \phi_{1}}{d t} \mid Z\right\rangle=-\langle u \nabla Y \mid Z\rangle+\kappa\left\langle\nabla^{2} Y \mid Z\right\rangle .
$$

The conditional averages in (4.2) can be evaluated by homogeneity of the velocity and scalar fields and Gaussianity of the reference fields

$$
\begin{aligned}
& \langle u \nabla Y \mid Z\rangle=0, \\
& \left\langle\frac{d \phi_{1}}{d t} \mid Z\right\rangle=\frac{\phi_{1}}{2\left\langle\phi_{1}^{2}\right\rangle} \frac{d\left\langle\phi_{1}^{2}\right\rangle}{d t}, \\
& \left\langle\nabla^{2} Y \mid Z\right\rangle=-\phi_{0} \frac{\left\langle\phi_{0 x}^{2}\right\rangle}{\left\langle\phi_{0}^{2}\right\rangle} \frac{\partial Y}{\partial \phi_{0}}+\left\langle\phi_{0 x}^{2}\right\rangle \frac{\partial^{2} Y}{\partial \phi_{0}^{2}}
\end{aligned}
$$




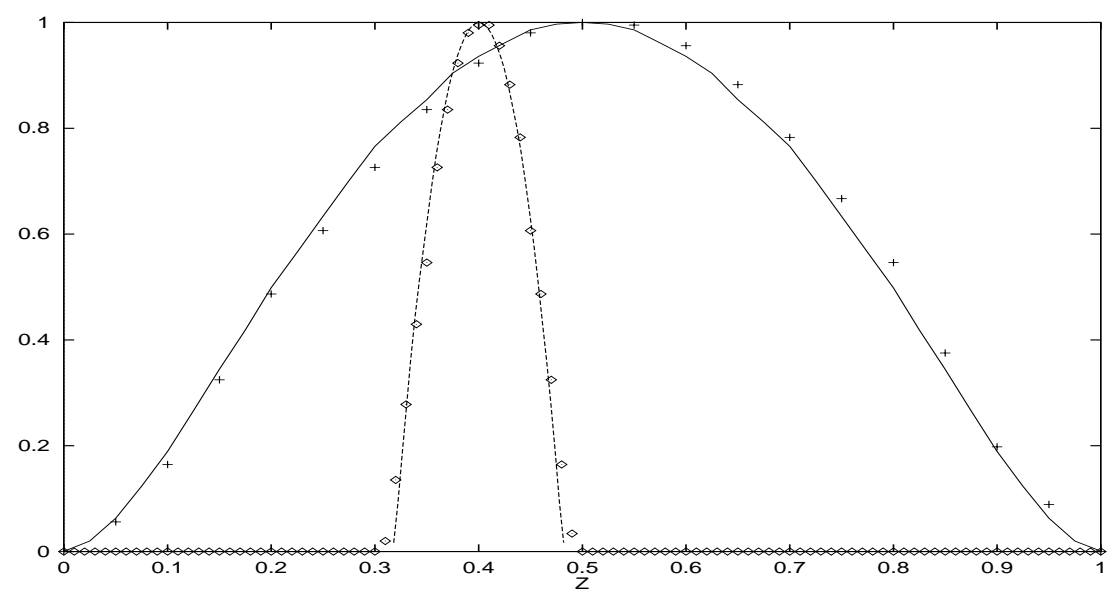

FIG. 4.1. Normalized $C D R s \chi(Z, t) / F(t)$ for the initially unsymmetric double-delta distribution with $A=0.6$. Line (DNS) and plus (model): initial CDRs; Dash (DNS) and diamond (model): final CDRs.

An exact solution can be obtained from (4.2) with the evaluated conditional averages (4.3) and the initial condition (2.5) which requires $\phi_{1}(0)=0$

$$
Y=\frac{1}{2}\left[1+\operatorname{erf}\left(\frac{\phi_{0}-\gamma e^{\tau}}{\sqrt{2} \Sigma}\right)+\left(1-\exp \left[\phi_{1}-\left\langle\phi_{1}\right\rangle+\int_{0}^{t}\left\langle\frac{d \phi_{1}}{d t} \mid Z\right\rangle d t\right]\right)(2\langle Z\rangle-1)\right]
$$

We will calculate the CDR $\chi(Z, t)=\kappa\left\langle(\nabla Y)^{2} \mid Z\right\rangle$, where ensemble average is taken over the level surface on which $\left(\phi_{0}, \phi_{1}\right)$ satisfies $Y\left(\phi_{0}, \phi_{1} ; t\right)=Z$. The expression obtained is then simplified by the first-order approximation of its Taylor expansion with respect to the mean $\left\langle\phi_{1}(t)\right\rangle$. This leads to

$$
\chi(Z, t)=F(t) \exp \left[-2\left(\operatorname{erf}^{-1}\left[(2 Z-1)-\left(1-\frac{1}{\sqrt{1+f_{\phi_{1}} t}}\right)(2\langle Z\rangle-1)\right]\right)^{2}\right]
$$

where

$$
\begin{aligned}
& F(t)=\frac{f_{\phi_{0}}}{\sqrt{2 \pi} \Sigma}, \\
& f_{\phi_{0}}=\kappa \frac{\left\langle\left(\phi_{x 0}\right)^{2}\right\rangle}{\left\langle\phi_{0}^{2}\right\rangle}, \\
& f_{\phi_{1}}=\frac{d\left\langle\phi_{1}^{2}\right\rangle}{d t} /\left\langle\phi_{1}^{2}\right\rangle .
\end{aligned}
$$

The time scales $f_{\phi_{0}}$ and $f_{\phi_{1}}$ are parameters of the present closure which must be input externally. Usually, they are reset at each time using a dynamical scheme, such as $f_{\phi_{0}}=\kappa\left\langle(\nabla Z)^{2}\right\rangle /\left\langle Z^{2}\right\rangle$ and $f_{\phi_{1}}=f_{\phi_{0}}$, so as to give correct evolution of the CDR. The CDR model (4.5) is compared with DNS of the diffusion equation with $\kappa=1$. In the case of homogeneity, the use of the diffusion equation to validate the CDR model for turbulent mixing has a reasonable justification. Figure 4.1 shows the initial and final CDRs obtained by 
the DNS and the CDR model (4.5) for the initially unsymmetric binary scalar with mean $\langle Z\rangle=0.4$. For exhibiting the shift of the maximum, the CDRs are normalized by their amplitudes $F(t)$ and (4.5) is rescaled by the scalar's variances.

The CDR model (4.5) is no longer separable in $Z$ and $t$. Its shape will shift while its amplitude decays with time. It is easy to verify $\chi(0.5,0) / F(t)=1$ and $\chi(\langle Z\rangle, \infty) / F(t)=1$ so that the location of maximum dissipation shifts from initial interface $Z=0.5$ to the scalar's mean $Z=\langle Z\rangle$. While $\langle Z\rangle=0.5$, the equation (4.5) recovers the CDR model (2.8) from the amplitude mapping closure. Therefore, the CDR model (4.5) correctly describes asymptotic behaviors of CDR: the CDR's maximum asymptotically approaches $Z=\langle Z\rangle$, while initially being at $Z=0.5$. We emphasize that (4.5) is the first term of the Taylor expansion of the CDR obtained from (4.4) with respect to the mean $\left\langle\phi_{1}(t)\right\rangle$. This simplified form gives the correct initial and asymptotic behavior of the unsymmetric binary mixing at the expense of an inaccurate description of the intermediate behavior. Indeed, it is well-known [9] that at short times, the CDR remains symmetric about the location of the initial interface. The symmetry point (location of maximum dissipation) begins to shift only after the elapse of a finite amount of time, depending on the extent of the initial asymmetry [14]. The present model predicts that the symmetry point begins to move immediately. A model which is also accurate at short times can be formulated from (4.4) in the general form

$$
\chi(Z, t)=F(t) \exp \left[-2\left(\operatorname{erf}^{-1}[(2 Z-1)-(1-f(t))(2\langle Z\rangle-1)]\right)^{2}\right]
$$

where $f(t)$ is a time-dependent function with $f(0)=1$ and $f(+\infty)=0$. The time-dependent function $f(t)$ is responsible for the shift of the CDR's shape. However, it is very difficult to write its expression explicitly, which needs a time scale as same as the case of amplitude function $F(t)$.

It has been shown that the mapping (4.1) is an appropriate approximation for a scalar gradient field. However, it is not expected that the mapping (4.1) makes the same accurate approximation to the scalar field as it does to the scalar gradient field itself. The reason is that a homogeneous random field is statistically orthogonal to its gradient field. The amplitude mapping (2.3) is an appropriate approximation to scalar fields but fails to approximate its gradient field. Generally speaking, the mapping closure carried out at the level of single-point PDFs is not valid for two-point PDFs such as gradient fields. This is the motivation to develop mapping closure approximation of higher orders. We expect that a higher-order approximation to a random field could produce a correct description of its gradient field.

5. Conclusion. We have developed a novel model of the conditional dissipation rate for turbulent mixing. The model is able to describe the asymptotic behavior of the CDR for either symmetric or unsymmetric initial double-delta distributions. The amplitude mapping closure has unsatisfactory asymptotic properties for the shift of the CDR's maximum. The problem can be solved using the extended mapping closure approximation developed in this paper. Further research is to account for the evolution of scalar variance by extending the CDR model (4.5). MCA can incorporate the effect of multiple time and length scales of practical interest in its predictions using more than one reference field. It provides a useful approach to describe statistics of turbulent mixing.

The author (G. W. He) is grateful for the very valuable discussions on the conditional dissipation rate with G. Kosaly and H. Pitsch. Special thanks are due to G. Kosaly for pointing out the exact solution (2.6). We thank S. S. Girimaji for his valuable comments on the shift process of the symmetric point. 


\section{REFERENCES}

[1] S. B. Pope, PDF methods for turbulent reactive flows, Progr. Energy. Combust. Sci., 11 (1985) pp. 119-192.

[2] P. J. Colucci, F. A. Jaberi, P. Givi, And S. B. Pope, Filtered density function for large eddy simulation of turbulent reacting flows, Phys. Fluids, 10 (1998), pp. 499-515.

[3] W. K. Bushe And H. Steiner, Conditional moment closure for large eddy simulation of nonpremixed turbulent reacting flows, Phys. Fluids, 11 (1999) pp. 1896-1906.

[4] H. Pitsch And H. Steiner, Large-eddy simulation of a turbulent piloted methane/air diffusion flame (Snadia flame D), Phys. Fluids, 12 (2000), pp. 2541-2554.

[5] R. H. Kraichnan, Closures for probability distribution, Bull. Am. Phys. Soc., 34 (1989), p. 2298.

[6] H. Chen, S. Chen, And R. H. Kraichnan, Probability distribution of a stochastically advected scalar field, Phys. Rev. Lett., 63 (1989) pp. 2657-2660.

[7] E. E. O'Brien AND T. L. JiAng, The conditional dissipation rate of an initial binary scalar in homogeneous turbulence, Phys. Fluids A, 3 (1991), pp. 3121-3123.

[8] E. E. O'Brien and A. SAhay, Asymptotic behavior of the amplitude mapping closure, Phys. Fluids A, 4 (1992), pp. 1773-1775.

[9] S. S. GIRIMAJI, A mapping closure for turbulent scalar mixing using a time-evolving reference field, Phys. Fluids A, 4 (1992), pp. 2875-2886.

[10] V. Eswaran and S. B. Pope, Direct numerical simulations of the turbulent mixing of a passive scalar, Phys. Fluids, 31 (1988), pp. 506-520.

[11] T. Gotoh and R. H. Kraichnan, Statistics of decaying Burgers turbulence, Phys. Fluids, 5 (1993), pp. $445-457$.

[12] Y. Kimura and R. H. Kraichnan, Statistics of an advected passive scalar, Phys. Fluids, 5 (1993), pp. 2264-2277.

[13] S. T. Orszag and L. R. Bissonnette, Dynamical properties of truncated Wiener-Hermite Expansions, Phys. Fluids, 10 (1967), pp. 2603-2613.

[14] T. L. JiAng, P. Givi And F. GaO, Binary and trinary scalar mixing by Fickian diffusion - Some mapping closure results, Phys. Fluids A, 4 (1992), pp. 1028-1035. 